\title{
Practice guidelines of the European Society of Hypertension for clinic, ambulatory and self blood pressure measurement
}

\author{
Eoin O'Brien, Roland Asmar, Lawrie Beilin, Yutaka Imai, Giuseppe Mancia, \\ Thomas Mengden, Martin Myers, Paul Padfield, Paolo Palatini, \\ Gianfranco Parati, Thomas Pickering, Josep Redon, Jan Staessen, \\ George Stergiou and Paolo Verdecchia on behalf of the European \\ Society of Hypertension Working Group on Blood Pressure Monitoring
}

Journal of Hypertension 2005, 23:697-701

Keywords: ambulatory blood pressure measurement, clinic blood pressure measurement, European Society of Hypertension, guidelines, self blood pressure measurement, sphygmomanometer

For affiliations, see Appendix.
Correspondence and requests for reprints to Professor Eoin O'Brien. Beaumont Hospital and Department of Clinical Pharmacology, Royal College of Surgeons, Beaumont Road, Dublin 9, Ireland.

Tel: +35312803 865; fax: +35312803 688; e-mail: eobrien@iol.ie

Received 6 December 2004 Accepted 10 December 2004

\section{Introduction}

Blood pressure measurement is the basis for the diagnosis, management, treatment, epidemiology and research of hypertension, and the decisions affecting these aspects of hypertension will be influenced, for better or worse, by the accuracy of measurement. An accurate blood pressure reading is a prerequisite, therefore, regardless of which technique is used, yet all too often the accuracy of measurement is taken for granted or ignored. This paper is a summary up-dated version of the recommendations published by the European Society of Hypertension (ESH) Working Group on Blood Pressure Monitoring for conventional (CBPM), ambulatory (ABPM) and self (SBPM) blood pressure measurement in 2003 ([1], from where further detailed information can be obtained).

\section{Aspects of blood pressure measurement common to all techniques \\ Factors affecting the technique \\ Selecting an accurate device \\ An accurate device is fundamental to all blood pressure measurement techniques. All devices used for blood pressure measurement should be subjected to indepen- dent evaluation according to one of the recognized pro- tocols. Details of devices and their validation status can be obtained on www.dableducational.org, a website devoted to blood pressure measurement. This website links to others (such as the British Hypertension Society - www.bhsoc.org) that also give information on blood pressure measuring devices.}

\section{Variability of blood pressure}

No matter which measurement device is used, blood pressure will always be a variable haemodynamic phenomenon that is influenced by many factors, which include the circumstances of measurement itself, emotion, exercise, meals, tobacco, alcohol, temperature, respiration, bladder distension, and pain; blood pressure is also influenced by age, race and diurnal variation, usually being lowest during sleep.

White-coat hypertension (isolated clinic hypertension)

White-coat hypertension is a condition in which an individual is hypertensive during repeated CBPM, but pressures measured outside the medical environment by ABPM or SBPM techniques are normal.

\section{White-coat effect}

This is the term used to describe the phenomenon found in many hypertensive patients whereby CBPM is usually greater than the blood pressure values obtained outside the clinic environment with ABPM or SBPM, the levels of which are nonetheless increased above normal.

Masked hypertension (isolated ambulatory hypertension) This phenomenon refers to patients in whom CBPM is normal but ABPM or SBPM are increased; in other words, hypertension is hidden until ABPM or SBPM is performed.

\section{The procedure}

Explanation to the patient

The first step in blood pressure measurement is adequate explanation of the procedure in an attempt to allay fear and anxiety, especially in nervous individuals.

\section{Attitude of observer}

Before taking the blood pressure, the observer should be in a comfortable and relaxed position, and should not rush 
the procedure, otherwise the cuff may be deflated too rapidly, resulting in underestimation of systolic and overestimation of diastolic pressures.

\section{Attitude of patient}

Patients should be encouraged to relax and be advised that neither they nor the observer should talk to each other for the few minutes before and during the blood pressure measurement.

\section{Posture of subject}

Blood pressure should be measured with the individual sitting with back support, legs uncrossed and the arm supported at heart level. Some patients may exhibit postural hypotension, especially with certain antihypertensive drugs. When this is likely, blood pressure should be measured with the patient standing.

\section{Which arm?}

Bilateral measurement should be made on first consultation and, if persistent differences greater than $20 \mathrm{mmHg}$ for systolic or $10 \mathrm{mmHg}$ for diastolic pressure are present on consecutive readings, the patient should be referred to a cardiovascular centre for further evaluation with simultaneous bilateral measurement and the exclusion of arterial disease.

\section{The cuff and bladder}

However sophisticated a blood pressure measuring device may be, if it is dependent on cuff occlusion of the arm (as are the majority of devices), it will then be prone to the inaccuracy induced by miscuffing, whereby a cuff contains a bladder that is either too long or too short relative to arm circumference (Table 1).

\section{Factors common to the patient}

Certain groups of people merit special consideration for blood pressure measurement. These include children; the elderly, who often have isolated systolic hypertension or autonomic failure with postural hypotension; obese people in whom the inflatable bladder may be too small for the arm size, leading to 'cuff hypertension'; patients

Table 1 Recommended bladder dimensions for adults

British Hypertension Society

Standard cuff Bladder $12 \times 26 \mathrm{~cm}$ for the majority of adult arms

Large cuff $\quad$ Bladder $12 \times 40 \mathrm{~cm}$ for obese arms

Small cuff Bladder $12 \times 18 \mathrm{~cm}$ for lean adult arms and children

American Heart Association

Small adult cuff

Bladder $10 \times 24 \mathrm{~cm}$ for arm circumference $22-26 \mathrm{~cm}$

Adult cuff

Bladder13 $\times 30 \mathrm{~cm}$ for arm circumference $27-34 \mathrm{~cm}$

Large adult cuff

Bladder16 $\times 38 \mathrm{~cm}$ for arm circumference $35-44 \mathrm{~cm}$

Adult thigh cuff Bladder $20 \times 42 \mathrm{~cm}$ for arm circumference $45-52 \mathrm{~cm}$ with arrhythmias in whom blood pressure measurement may be difficult and the mean of a number of measurements may have to be estimated; pregnant women in whom the disappearance of sounds (phase V) is the most accurate measurement of diastolic pressure, except when sounds persist to zero, when the fourth phase of muffling of sounds should be used; and any individual during exercise.

\section{Clinic (conventional/office) sphygmomanometry \\ Basic requirements for auscultatory blood pressure measurement}

The accurate measurement of blood pressure in clinical practice by the century-old technique of Riva Rocci/ Korotkoff is dependent on the individual, the equipment used and the observer. Errors in measurement can occur at each of these points of interaction of the technique, but by far the most fallible component is the observer.

\section{Observer error}

Observer error, which can greatly affect the accuracy of measurement, may be the result of systematic error, terminal digit preference or observer prejudice or bias.

\section{Mercury and aneroid sphygmomanometers}

The mercury sphygmomanometer is a reliable device, but all too often its continuing efficiency has been taken for granted, whereas the aneroid manometer is not generally as accurate. An inflation/deflation system, an occluding bladder encased in a cuff, and auscultation using a stethoscope are features common to these devices, any of which may introduce error. Users should be aware of the hazards of mercury, which may soon be banned from use in clinical medicine. Aneroid sphygmomanometers register pressure through a bellows and lever system, which may become inaccurate with everyday use, usually leading to false low readings with the consequent underestimation of blood pressure. A stethoscope should be of a high quality, with clean, well-fitting earpieces.

\section{Automated devices as alternatives to the mercury sphygmomanometer}

An accurate automated sphygmomanometer capable of providing printouts of systolic and diastolic blood pressure, together with heart rate and the time and date of measurement, should eliminate errors of interpretation and abolish observer bias and terminal digit preference, and should be used when possible. Moreover, the need for elaborate training would no longer be necessary, although a period of instruction and assessment of proficiency in using the automated device will always be necessary.

\section{Performing auscultatory measurement}

- The observer should ensure that the manometer is no more than $1 \mathrm{~m}$ away so that the scale can be read easily, 
that the mercury column is vertical, that the bladder dimensions are accurate, and that, if the bladder does not completely encircle the arm, its centre is over the brachial artery.

- The stethoscope should be placed gently over the brachial artery at the point of maximal pulsation; the cuff should then be inflated rapidly to about $30 \mathrm{mmHg}$ above the palpated systolic pressure and deflated at a rate of 2-3 $\mathrm{mmHg}$ per pulse beat (or per second), during which the Korotkoff sounds will be heard.

- Systolic blood pressure is the first appearance of faint, repetitive, clear tapping sounds that gradually increase in intensity.

- Diastolic blood pressure is at the point of disappearance of sounds, or the first $\mathrm{mmHg}$ value at which the sounds are no longer audible (phase V); when sounds persist down to zero, muffling of sounds (phase IV) should be recorded for diastolic pressure.

\section{Ambulatory blood pressure measurement}

The technique of ABPM is being used increasingly in clinical practice.

\section{General considerations Advantages of ambulatory blood pressure measurement} ABPM has a number of advantages: it provides a profile of blood pressure away from the medical environment, thereby allowing identification of individuals with a white-coat response; it shows blood pressure behaviour over a 24-h period during usual daily activities, rather than when the individual is sitting in the artificial circumstances of a clinic or office; it can indicate the duration of decreased blood pressure over a 24 -h period; it can identify patients with blunted or absent blood pressure reduction at night - the non-dippers - who are at greater risk for organ damage and cardiovascular morbidity; it can demonstrate a number of patterns of blood pressure behaviour that may be relevant to clinical management, such as white-coat hypertension, isolated systolic hypertension, masked hypertension, hypotension, and enhanced blood pressure variability. In long-term outcome studies, ABPM has been shown to be a stronger predictor of cardiovascular morbidity and mortality than is CBPM.

\section{Choosing an ambulatory blood pressure monitoring device}

The website - www.dableducational.org - provides upto-date information on ABPM devices.

\section{Defining daytime and night-time periods}

There are a number of ways of analysing blood pressures recorded during the 24-h cycle. One simple and popular method of determining the time of awakening and sleeping is to assess them from diary card entries. Another method is to use a fixed-time method in which the retiring (2101-0059 h) and rising (0601-0859 h) periods (during which blood pressures are subject to considerable variation) are eliminated, with the daytime period being from 0900 to $2100 \mathrm{~h}$ and night-time from 0100 to $0600 \mathrm{~h}$; in this way the variations that may exist between the young and the old and in different cultures are to some extent eliminated from the analysis. This method has the disadvantage of eliminating information on the white-coat window and earlymorning surge of blood pressure, which may be associated with cardiovascular events. However, these windows can be analysed separately for research or if considered important in practice.

\section{Technique}

In clinical practice, measurements are usually made at 20- to 30-min intervals in order not to interfere with activity during the day and with sleep at night, but measurements can be made more frequently when indicated. Whatever definition of daytime and nighttime is used, at least two-thirds of systolic and diastolic blood pressures during the daytime and night-time periods should be satisfactory, and if this minimum requirement is not met, the ABPM should be repeated. To improve reproducibility, ABPM measurements should be performed on like days, for example on working (most commonly) or recreational days. A diary card may be used to record symptoms and events that may influence ABPM measurements, in addition to the time of drug ingestion, meals and going to and arising from bed. If there are sufficient measurements, editing is not necessary for calculating average 24-h, daytime, and night-time values, and only grossly incorrect readings should be deleted from the recording.

\section{Definition of normality}

Normal ranges for ABPM are average daytime ABPM of less than $135 / 85 \mathrm{mmHg}$ and average night-time ABPM less than $120 / 70 \mathrm{mmHg}$, but even lower values are advocated, particularly in high-risk groups such as diabetic patients (Table 2). Normal ranges in pregnancy are also available.

Table 2 Recommended levels of normality for ambulatory blood pressure monitoring in adults

\begin{tabular}{llll}
\hline & \multicolumn{3}{c}{ Blood pressure value $(\mathrm{mmHg})$} \\
\cline { 2 - 4 } & Optimal & Normal & Abnormal \\
\hline Awake & $<130 / 80$ & $<135 / 85$ & $>140 / 90$ \\
Asleep & $<115 / 65$ & $<120 / 70$ & $>125 / 75$ \\
\hline
\end{tabular}

The evidence supporting the normal and abnormal demarcation values are based on a number of outcome studies; evidence is not yet available to make recommendations for the intermediate pressure ranges between the 'normal' and 'abnormal' values, or for recommendations lower than those given. It must be emphasized that these values are only a guide to 'normality', and that lower 'optimal' values may be more appropriate in patients whose total cardiovascular risk-factor profile is high, and in whom there is concomitant disease such as diabetes mellitus. 
Table 3 Clinical indications for ambulatory blood pressure monitoring

Accepted indications

Suspected white-coat hypertension

Suspected nocturnal hypertension

Suspected masked hypertension

To establish dipper status

Resistant hypertension

Hypertension of pregnancy

Potential indications

Elderly patient

As a guide to antihypertensive drug treatmen

Type 1 diabetes

Evaluation of symptoms suggesting

orthostatic hypotension

Autonomic failure

\section{Clinical indications}

Ambulatory blood pressure monitoring is accepted as being of benefit in patients with the conditions listed in Table 3.

\section{Who should be re-monitored?}

In individuals with increased CBPM and normal $\mathrm{ABPM}$ - that is, white-coat hypertension - it is advisable to confirm the diagnosis by repeating ABPM within 3-6 months. In individuals with confirmed white-coat hypertension and a normal risk-factor profile, ABPM should be repeated annually, or every 2 years if the pattern appears to be established and consistent, as is often the case. SBPM may be combined with ABPM to reduce the frequency of $A B P M$. The frequency of repeat ABPM to evaluate the efficacy of antihypertensive medication will be dependent on the severity of hypertension, and the response to treatment. These recommendations are arbitrary, and the decision as to when to repeat ABPM must be based on clinical indications for individual patients.

\section{Self blood pressure measurement}

Self blood pressure measurement is popular among patients, as indicated by the huge sales of devices. Although there is a revival of interest in this old methodology, based on evidence suggesting that the regular use of SBPM under medical guidance may help to achieve better control of blood pressure, there is a need for further research to determine the precise role of SBPM in practice.

\section{General considerations \\ Devices and validation}

The types of monitor available for SBPM include mercury column sphygmomanometers and aneroid manometers, but electronic devices using oscillometry are becoming more popular and are replacing the auscultatory technique. These devices require less training and are more suitable for patients with infirmities such as arthritis and deafness. Freedom from medical control, coupled with a growing public desire to know more about health and illness, have resulted in the manufacture and marketing of a vast array of such devices, few of which have been evaluated according to the procedures considered necessary for blood pressure measuring equipment used in clinical practice. Devices that measure blood pressure at the finger are not recommended, because of the inaccuracies arising from distortion of measurements as a result of peripheral vasoconstriction, alterations in blood pressure at more distal sites of recording, and the effect of limb position on blood pressure. Devices that measure blood pressure at the wrist are subject to the latter two problems and, although they are more accurate than finger measuring devices, there are strong reservations about the correct use of these devices. If the wrist is not held at heart level during measurement, inaccurate measurements will be obtained, and measurement is also influenced by flexion and hyperextension of the wrist. Appropriate cuff sizes should be available. It may not be possible to measure blood pressure with automated devices in patients with arrhythmias, and there are some patients in whom, for no obvious reason, automated measurement is not possible. A major advantage of automated devices is a capability to store measurements, to provide hard copy of measurements and to transmit measurements electronically or telephonically. Considering that the number of devices for SBPM available on the market that have fulfilled independent validation criteria is small, the state of the market needs to be assessed regularly, with the results being easily accessible to prospective purchasers. The website devoted to blood pressure measurement www.dableducational.org - can be consulted to determine which devices have been validated.

\section{User procedure}

The recommendations for SBPM do not vary in principle from those that apply for blood pressure measurement in general, but there are some points in need of emphasis. The procedure should be performed under medical supervision; a period of rest should precede each measurement; brachial artery occluding devices are preferred to wrist monitors; memory-equipped devices, which can store or transmit data, have beneficial advantages; in the initial phase of assessment or when treatment is being initiated, two measurements in the morning and two measurements in the evening are recommended each day for 1 week, and for long-term observation measurements, this routine can be reduced to 1 week per quarter.

\section{Diagnostic thresholds}

The threshold level of 135/85 mmHg (130/80 mmHg optimal) for SBPM is the same as that for mean daytime $\mathrm{ABPM}$, and should be the average of several readings taken over several days. SBPM needs to be further evaluated in prospective outcome studies.

\section{Supervision}

At present, SBPM is performed mostly by patients on their own initiative, using devices bought on the free market, without medical control; this is undesirable and the procedure should be performed under medical 
supervision, by patients who have been instructed in the technique. Primary-care physicians should see SBPM as a means of gaining further insight into blood pressure control and the effects of management strategies in motivated and informed patients who remain under medical supervision.

\section{Clinical indications}

The clinical applications of SBPM are only beginning to become apparent as the technique becomes more widely used and scientific data are gathered, but these are broadly similar to those for ABPM. There is some evidence that SBPM may improve compliance with antihypertensive medication.

\section{Reference}

1 O'Brien E, Asmar R, Beilin L, Imai Y, Mallion JM, Mancia G, et al. European Society of Hypertension recommendations for conventional, ambulatory and home blood pressure measurement. European Society of Hypertension Working Group on Blood Pressure Monitoring [Review]. J Hypertens 2003; 21:821-848.

\section{Appendix}

Membership of the ESH working group on blood pressure monitoring

\section{Australia}

Lawrie Beilin, Royal Perth Hospital Unit, Perth.

\section{Belgium}

Denis L. Glement, Universitair Ziekenhuis, Gent; Robert Fagard, Jan Staessen, Katholieke Universiteit Leuven, Leuven.

\section{Canada}

Martin G. Myers, Sunnybrook and Women's College Health Sciences Centre, Toronto.

\section{France}

Jean-Michel Mallion, Centre Hospitalier Universitaire de Grenoble, Grenoble; Roland Asmar, Société Française d'Hypertension Arterérielle, Paris.

\section{Germany}

Thomas Mengden, University Clinic Bonn, Bonn.

\section{Greece}

George Stergiou, Third University Department of Medicine, Athens.

Ireland

Eoin O'Brien (Chairman), Beaumont Hospital, Dublin.

\section{Italy}

Giuseppe Mancia, Universita Degli Studi di MilanoBicocca, Monza; Gianfranco Parati, University of
Milano-Bicocca and Ospedale San Luca, Milan; Paolo Palatini, Universita' di Padova, Padua; Paolo Verdecchia, Ospedale R. Silvestrini, Perugia.

\section{Japan}

Yutaka Imai, Tohoku University Graduate School of Pharmaceutical Science and Medicine, Sendai.

Spain

Josep Redon, University of Valencia, Valencia.

\section{Switzerland}

Bernard Waeber, Centre Hospitalier Universitaire Vaudois, Lausanne.

\section{The Netherlands}

Gert van Montfrans, Academisch Medisch Centrum, Amsterdam; Peter De Leeuw, Academisch Ziekenhuis, Maastricht.

\section{United Kingdom}

Paul L. Padfield, Western General Hospital, Edinburgh.

USA

William White, The University of Connecticut Health Center, Farmington, Connecticut; Thomas G. Pickering, Columbia University College of Physicians and Surgeons, New York. 